\title{
Carrier-envelope phase effects on the strong-field photoemission of electrons from metallic nanostructures
}

\author{
Björn Piglosiewicz ${ }^{1,2}$, Slawa Schmidtt,2, Doo Jae Park ${ }^{1,2}$, Jan Vogelsang, ${ }^{1,2}$, Petra Groß ${ }^{1,2}$, \\ Cristian Manzoni ${ }^{3}$, Paolo Farinello ${ }^{3}$, Giulio Cerullo ${ }^{3}$ and Christoph Lienau ${ }^{1,2 \star}$
}

\begin{abstract}
Sharp metallic nanotapers irradiated with few-cycle laser pulses are emerging as a source of highly confined coherent electron wave packets with attosecond duration and strong directivity ${ }^{1-6}$. The possibility to steer, control or switch such electron wave packets with light ${ }^{7}$ is expected to pave the way towards direct visualization of nanoplasmonic field dynamics $^{8-10}$ and real-time probing of electron motion ${ }^{11,12}$ in solid-state nanostructures ${ }^{13,14}$. Such pulses can be generated by strong-field-induced tunnelling and acceleration of electrons in the near-field of sharp gold tapers within one half-cycle of the driving laser field ${ }^{1,2,5}$. Here, we show the effect of the carrier-envelope phase of the laser field on the generation and motion of strong-field-emitted electrons from such tips. We observe clear variations in the width of plateau-like photoelectron spectra characteristic of the subcycle regime. This is a step towards controlling the coherent electron motion in and around metallic nanostructures over ultrashort lengths and timescales.
\end{abstract}

During the last two decades, strong-field effects in the interaction of light with atomic and molecular systems have led to a wealth of new physical phenomena, including the emission of high-harmonic radiation $^{15,16}$ and the generation of attosecond light and X-ray pulses ${ }^{17}$. Key to those phenomena is a field-induced periodic modulation of the tunnelling barrier, acceleration of the photoemitted electrons within an essentially spatially homogeneous laser field, and their recollision with the nuclei ${ }^{18}$. This has opened up the possibility to image molecular wavefunctions ${ }^{12}$ and to probe electronic motion in real time. Attosecond science is enabled by the capability to control the carrier-envelope phase (CEP) of the light pulse, giving access to the electric-field waveform rather than the intensity profile.

More recently, related strong-field photoemission phenomena have been demonstrated for metallic nanostructures ${ }^{1,4,5,19,20}$. For metallic tapers with modest field enhancement, for example, tungsten tips, multiphoton ionization (MPI) ${ }^{2,6}$ and above-threshold ionization (ATI $)^{4,20}$ have been observed. CEP effects in this regime are ascribed to changes in the interference pattern created by electrons emitted in subsequent cycles, while the effect of the electric field amplitude is weak ${ }^{4}$. In contrast, for gold tips with large field enhancement, optical field-induced tunnelling becomes important $t^{4,5,21}$ and, for sharp tips with pronounced spatial field gradients, a new subcycle emission regime has recently been discovered ${ }^{1}$. In this new regime, the electron dynamics are fundamentally different from those known in atomic and molecular systems because the decay length of the optical near-field $l_{\mathrm{F}}$ is shorter than the quiver amplitude $l_{\mathrm{q}}$ of the electrons $\left(\delta=l_{\mathrm{F}} / l_{\mathrm{q}}<1\right)$. Hence, the field-emitted electrons are highly directionally accelerated ${ }^{5}$, escaping the local near-field within less than half an optical cycle (for an overview of the different regimes, see Supplementary Section 3). Here, we study for the first time the influence of the CEP of the driving field on the generation and motion of strong-field-emitted electrons from such sharp gold tips. When illuminating ultra-sharp nanometre-sized gold tapers with few-cycle near-infrared pulses, we observe a sensitive dependence of the electron acceleration dynamics on the CEP. Control of electron motion by the phase of a strong light field, which is of key importance for coherent control of high-harmonic generation $(\mathrm{HHG})^{16,21,22}$ and attosecond streaking ${ }^{7,23,24}$ in gases, is here extended to single solid-state nanostructures.

We study photoemission from sharply etched, single-crystalline gold tapers ${ }^{25}$. These tapers have a radius of curvature as low as $5 \mathrm{~nm}$ and are characterized by a large optical field enhancement factor of $\sim 9$ and a short decay length of the local near-field at the taper apex of $\sim 2 \mathrm{~nm}$ (ref. 5). Few-cycle near-infrared pulses are generated by difference frequency mixing of the outputs from two independently tunable non-collinear optical parametric amplifiers (NOPAs) ${ }^{26}$. The set-up provides passively CEP-stabilized pulses with a centre wavelength tunable between 1.3 and $1.9 \mu \mathrm{m}$. Their duration of $14 \mathrm{fs}$, corresponding to 2.6 cycles at $1.65 \mu \mathrm{m}$, is measured by interferometric frequency-resolved second-harmonic autocorrelation (Fig. 1b). The pulses display a high CEP stability with fluctuations of $66 \mathrm{mrad}$ (root-mean-square) over a $600 \mathrm{~s}$ interval (Supplementary Section 2). The pulses, with their CEP controlled via a pair of fused silica wedges (Fig. 1c), are tightly focused to a diameter of $\sim 2 \mu \mathrm{m}$ on the gold tips by an all-reflective objective $^{27}$. We first investigated the effect of laser intensity on the electron yield using incident pulses of up to $700 \mathrm{pJ}$ energy (Fig. 2a). Assuming a field enhancement factor of $f=9$ (ref. 5), the peak intensity at the tip is then varied from 0 to $f^{2} \cdot I=74 \mathrm{TW} \mathrm{cm}^{-2}$, corresponding to a local peak field strength $f \cdot E_{0}$ at the tip apex of up to $23.6 \mathrm{~V} \mathrm{~nm}^{-1}$. At low intensities we observe a steep nonlinear increase in yield that is characteristic of $\mathrm{MPI}^{6}$. The order of nonlinearity of $\sim 7.5$ matches well with an estimated workfunction of gold of $\Phi=5.5 \mathrm{eV}$ and an average photon energy of $0.7 \mathrm{eV}(\lambda=1.65 \mu \mathrm{m})$. At higher intensities above $15 \mathrm{TW} \mathrm{cm}^{-2}$, the order of nonlinearity begins to decrease and saturation of the yield is observed. This is one of the signatures of the transition from MPI into a strong-field emission regime ${ }^{19}$. This transition appears for a Keldysh parameter $\gamma=\left(\sqrt{\Phi} / \sqrt{2 U_{\mathrm{p}}}\right)$ of $\sim 0.9$, where $U_{\mathrm{p}}$ is the ponderomotive energy $U_{\mathrm{p}}=$ $\left(\left(e f E_{0}\right)^{2} / 4 m \omega^{2}\right)$. The transition is accompanied by a distinct change of the corresponding photoelectron spectra. The narrow emission spectra characteristic of MPI broaden considerably with increasing intensity until we see an essentially plateau-like kinetic

Institut für Physik, Carl von Ossietzky Universität, 26129 Oldenburg, Germany, ${ }^{2}$ Center of Interface Science, Carl von Ossietzky Universität, 26129 Oldenburg, Germany, ${ }^{3}$ IFN-CNR, Dipartimento di Fisica, Politecnico di Milano, 20133 Milano, Italy. *e-mail: christoph.lienau@uni-oldenburg.de 

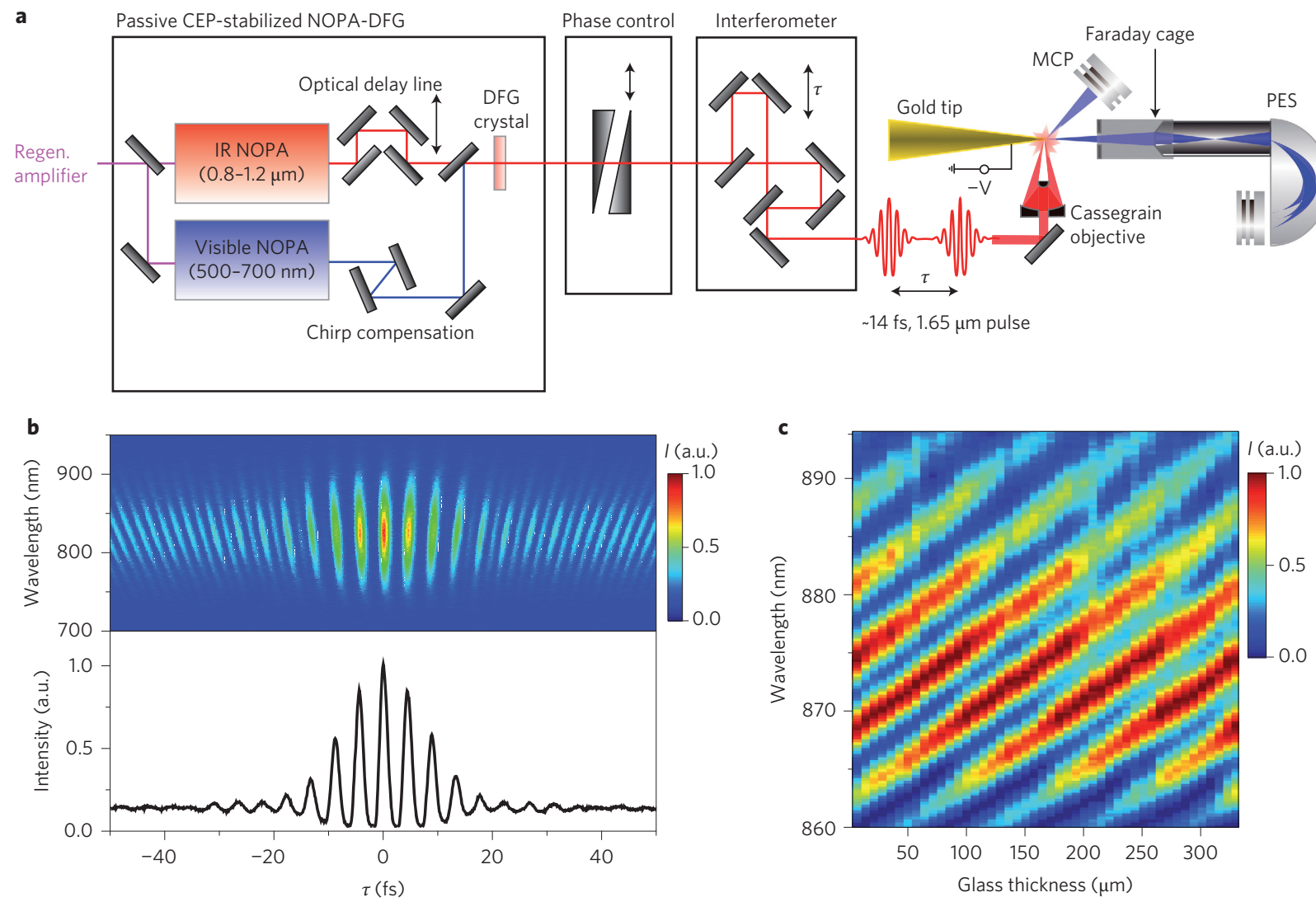

Figure 1 | Electron generation from sharp metal tips with few-cycle CEP-stabilized pulses. a, Generation of passively CEP-stabilized few-cycle near-infrared pulses by difference frequency generation (DFG) of pulses from two independently tunable NOPAs. CEP variation is achieved by wedge insertion, and a Michelson interferometer is used for peak intensity control and time-resolved measurements. The pulses are focused onto a sharply etched nanometre-sized gold tip to induce strong-field photoemission. The electron yield is measured by a microchannel plate (MCP), and kinetic energy spectra are recorded by a photoelectron spectrometer (PES). b. Frequency-resolved (top) and spectrally integrated (bottom) interferometric autocorrelation traces of the near-infrared NOPA pulses centred at $1.65 \mu \mathrm{m}$, indicating a pulse duration of $14 \mathrm{fs}$. c. Measurement of the CEP variation in an $f$-to- $2 f$ interferometer as a function of the insertion of a pair of fused-silica wedges.

energy spectrum (insets, Fig. 2a). This spectral shape is a definite signature of strong-field acceleration of photoemitted electrons within the steep near-field gradient at the tip apex ${ }^{1,5}$.

Simulations of the energy spectra within a modified threedimensional Simpleman model ${ }^{5}$ show that the energetic width of the plateau-like spectra is given roughly by $1.2 U_{\mathrm{p}}$. This provides a measure of the local field strength at the tip apex and, by comparison with the incident pulse intensity, we can estimate a field enhancement of $f \approx 9$ (Supplementary Section 5). In these simulations we assume that electrons are generated only during negative cycles, $E(t)<0$. Electrons are emitted from a thin layer near the metal surface and are accelerated away from the tip ${ }^{28}$. We take an analytical model for the optical near-field distribution around the apex. The local generation yield is deduced from a FowlerNordheim equation, and the ejected electrons are accelerated as classical particles within the temporally oscillating and spatially varying near-field. We find that the energetic shape of the spectra depends sensitively on the decay length of the local near field $l_{\mathrm{F}}$ and on the quiver amplitude $l_{\mathrm{q}}=\sqrt{2 U_{\mathrm{p}} /\left(m \omega^{2}\right)}$ of the lightdriven electrons. Only in the subcycle regime, $\delta=l_{\mathrm{F}} / l_{\mathrm{q}}<1$, are electrons accelerated out of the near-field within less than one half-cycle ${ }^{1,5}$ and plateau-like spectra emerge (Fig. 2a, top left inset). From the width of the spectra we can therefore deduce both $U_{\mathrm{p}}$ and $l_{\mathrm{q}}$ and estimate a near-field decay length of $\sim 2 \mathrm{~nm}$ for our sharp gold tapers (Supplementary Section 5).

Interferometric autocorrelation (IAC) measurements, which detect the electron yield as a function of the time delay between incident pulses, show a few narrow emission spikes with a width less than $1 \mathrm{fs}$ (Fig. 2a, right inset). The measurements agree well with simulations assuming 2.6 cycle pulses and electron generation during negative half-cycles only. We also assumed that the generation yield follows the same intensity dependence as in Fig. 2a. The simulations indicate electron emission during the central two cycles of the laser pulses. Both the IAC measurements and the observation of plateau-like spectra can be taken as an indication of a prompt release of electrons from the metal tip. A temporally delayed emission process, possibly due to fast electron relaxation, would result in substantial changes to the spectral shape.

We now study the effect of CEP variations on the kinetic energy spectra in the transition region between MPI and strong-field emission, for a local field strength of $f \cdot E_{0} \approx 11 \mathrm{~V} \mathrm{~nm}^{-1}$. Figure $2 \mathrm{~b}$ presents a series of 60 electron spectra recorded while varying the CEP by more than $6 \pi$. In this regime we find pronounced variations of the total electron count rate of $\sim 50 \%$, while the spectral shape remains unaffected. This may be ascribed to the strong nonlinearity of the electron yield when assuming that electrons are released during negative half-cycles only.

Very different CEP effects are seen in the strong-field regime. CEP-dependent spectra were recorded with $16 \mathrm{fs}$ pulses with a higher local field amplitude of $f \cdot E_{0} \approx 15 \mathrm{~V} \mathrm{~nm}^{-1}$ (Fig. 3a). In this regime we find that the effect of the CEP on electron yield is much less pronounced, reflecting the saturation of the electron yield. Instead, marked variations of the high- and low-energy cutoffs of the plateau-like spectra are observed. Comparing 
a

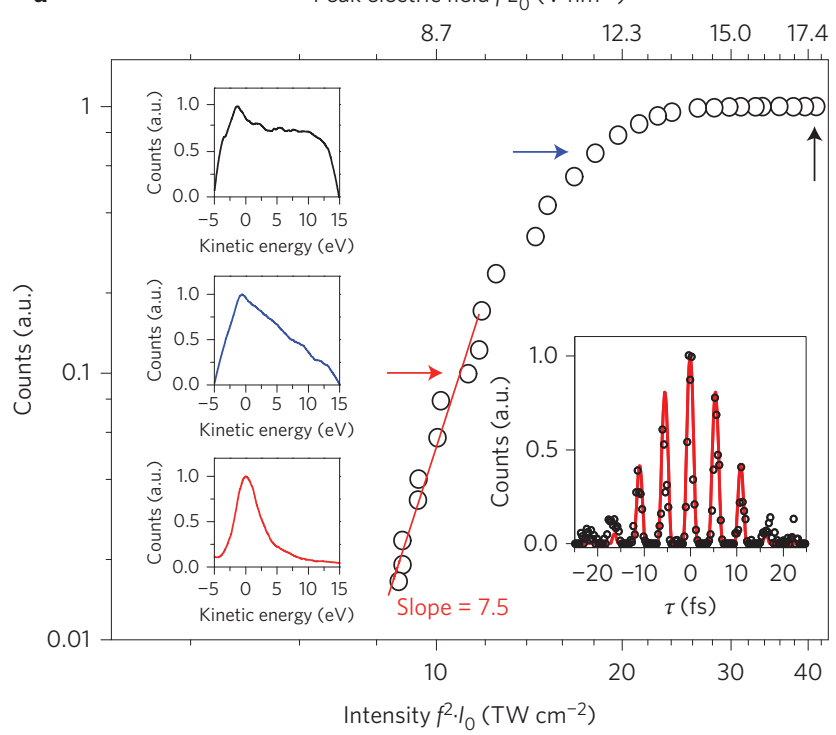

b

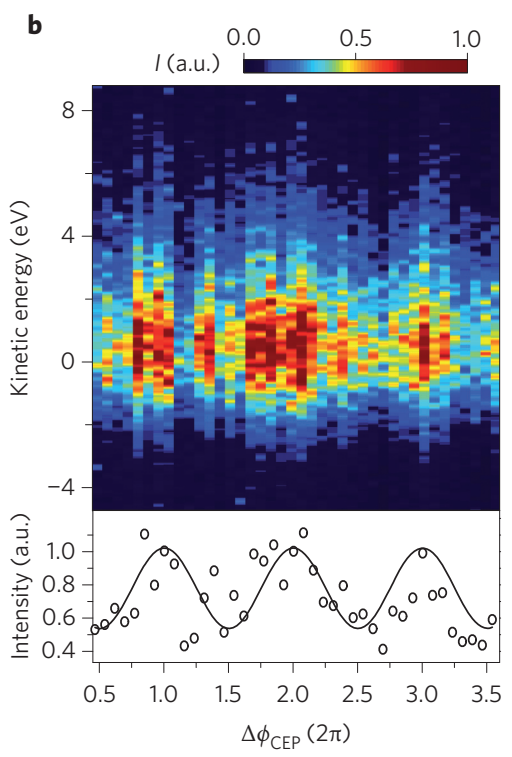

Figure 2 | Transition from multiphoton to strong-field photoemission from sharp gold tips. a, Electron yield as a function of intensity (bottom axis) and the corresponding peak electric field $f \cdot E_{0}$ (top axis) at the tip apex. For $f \cdot E_{0}<10 \mathrm{Vnm}^{-1}$, MPI dominates with a high nonlinearity of order $\sim 7.5$. For larger amplitudes, electron emission begins to saturate and is governed by optical-field-induced tunnelling. Corresponding kinetic energy spectra (black, blue and red insets) show a transition to a broad plateau-like spectrum in the strong-field regime. Bottom right inset: interferometric autocorrelation trace recorded by electron detection (black line) and simulation for a $16 \mathrm{fs}$ pulse at a $1.65 \mu \mathrm{m}$ centre wavelength with $f \cdot E_{0}=13 \mathrm{~V} \mathrm{~nm}^{-1}$ (red line). b, Effect of CEP variation on kinetic energy spectra, recorded with $14 \mathrm{fs}$ pulses at $1.6 \mu \mathrm{m}$ in the intermediate regime with $f \cdot E_{0}=11 \mathrm{~V} \mathrm{~nm}{ }^{-1}$. Clearly shown are pronounced variations in the electron yield (see energy-integrated curve) and the width of the spectra.

a
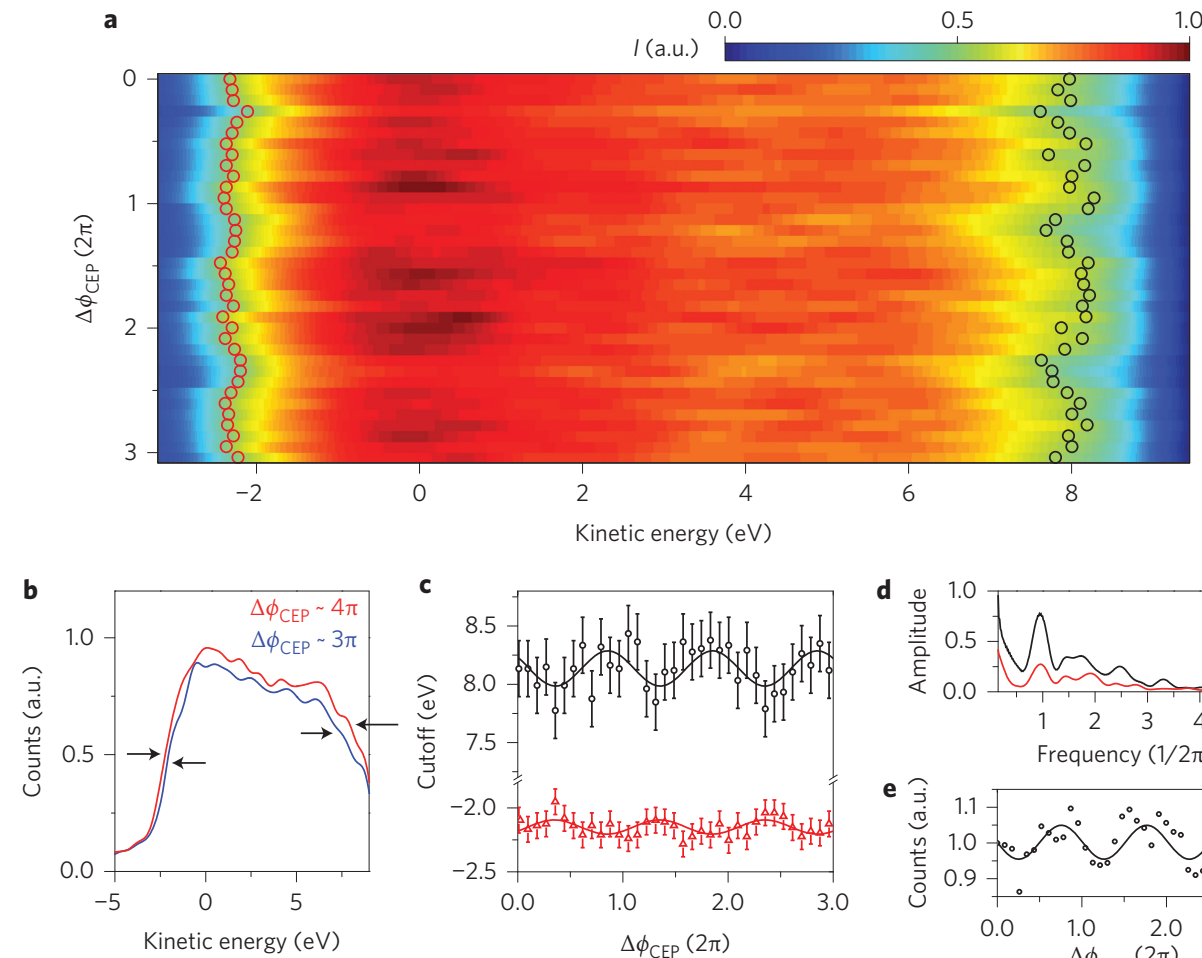

c
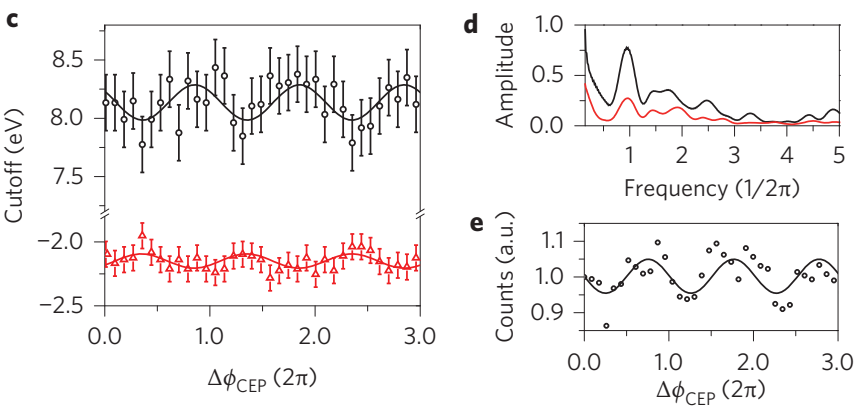

Figure 3 | Experimental observation of CEP effects in strong-field electron emission from sharp metal tips. a, Kinetic energy spectra recorded with 16 fs pulses at $1.65 \mu \mathrm{m}$ with $f \cdot E_{0}=15.4 \mathrm{~V} \mathrm{~nm}{ }^{-1}$ while varying the CEP by wedge insertion. A clear modulation of the spectral width with $2 \pi$ periodicity is observed. Red and black circles indicate low- and high-energy cutoffs, respectively. $\mathbf{b}$, Two representative kinetic energy spectra recorded at $\Delta \phi_{\text {CEP }} \approx 3 \pi$ (blue curve) and $4 \pi$ (red curve) demonstrate the spectral variation when changing the CEP by $\pi$. Black arrows indicate the low- and high-energy cutoffs. c, Experimental high (black squares) and low (red triangles) cutoff energies extracted from a and sine functions fitted to these values. Error bars originate from the standard deviation of electron counts in the kinetic energy spectra. $\mathbf{d}$, Fourier analysis of the experimental low- (red) and high- (black) energy cutoff values from c. e, Total electron yield $I\left(\phi_{\mathrm{CEP}}\right)=\int I\left(\phi_{\mathrm{CEP},} E_{\mathrm{kin}}\right) \mathrm{d} E_{\mathrm{kin}}$ determined by integration over the spectra in a, showing the same periodicity as the cutoff energy variation. 


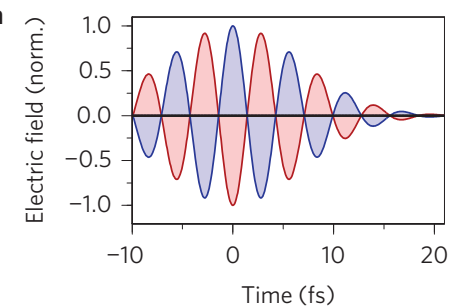

b

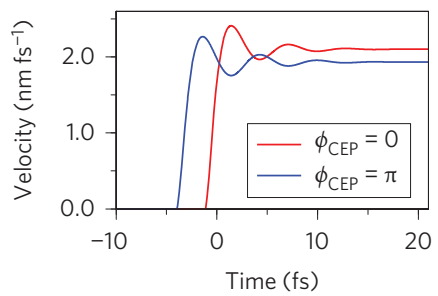

C

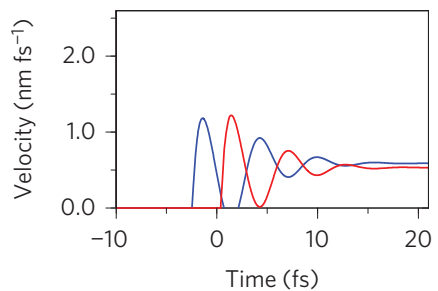

d

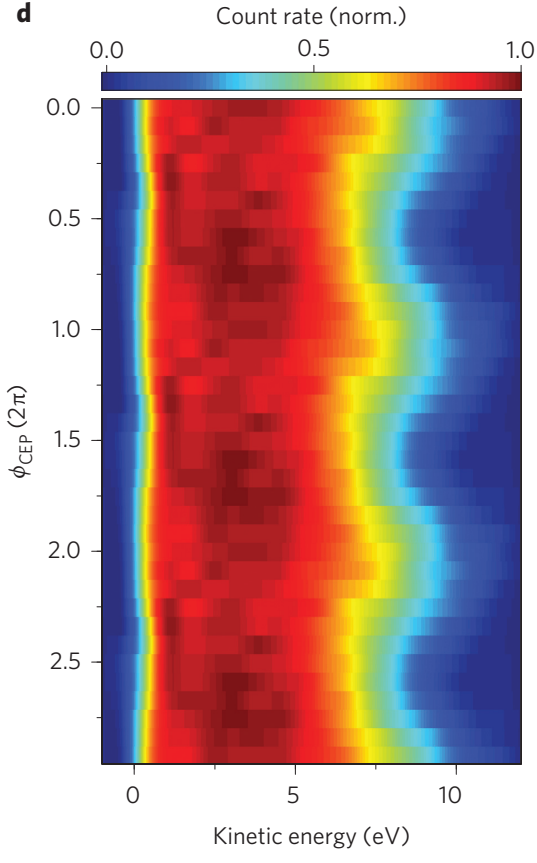

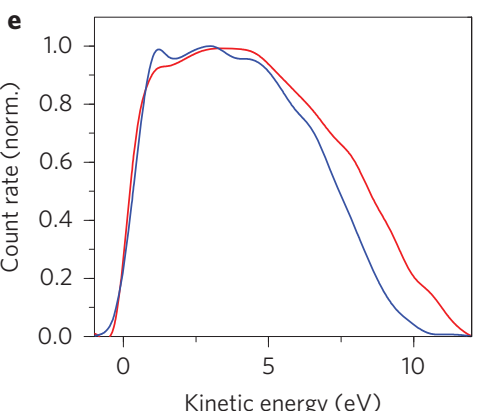

f

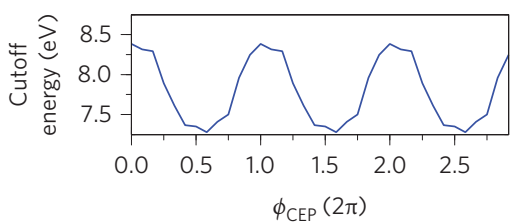

g

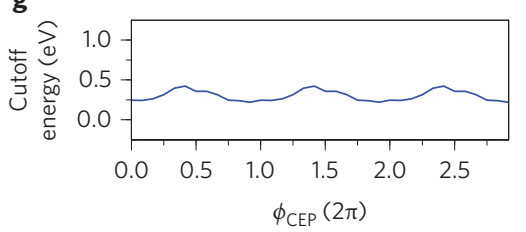

Figure 4 | Numerical simulation of the CEP effect on strong-field photoemission. $\mathbf{a}$, Electric field of a $16 \mathrm{fs}$ pulse centred at $1.65 \mu \mathrm{m}$ for $\phi_{\mathrm{CEP}}=0$ (red shaded area) and $\phi_{\mathrm{CEP}}=\pi$ (blue shaded area). $\mathbf{b}$, Time evolution of the velocity of subcycle electrons emitted at the beginning of the main cycle, that is, at $\Delta t_{\mathrm{B}}=-\pi / 2 \omega$ for $\phi_{\mathrm{CEP}}=0$ (red curve) and for $\phi_{\mathrm{CEP}}=\pi$ (blue curve). In both cases, the electrons are mainly accelerated during the initial half-cycle and no quiver motion is seen. c, Velocity evolution for two electrons emitted from pulses with $\phi_{\mathrm{CEP}}=0$ (red curve) and $\phi_{\mathrm{CEP}}=\pi$ (blue curve) but for $\Delta t_{\mathrm{B}}=0.1 \pi / \omega$. Acceleration is mainly gained during the second cycle. $\mathbf{d}$, Simulated kinetic energy spectra $I\left(\phi_{\mathrm{CEP},} E_{\mathrm{kin}}\right)$. e, Representative kinetic energy spectra, $I\left(0, E_{\mathrm{kin}}\right)$ and $I\left(\pi, E_{\text {kin }}\right)$, shown as red and blue curves, respectively. $\mathbf{f}$, High-energy cutoff extracted from $\mathbf{d}$, following a sine function, and $\mathbf{g}$, out-of-phase variation of the low-energy cutoff, resembling a saw-tooth function.

spectra for $\Delta \phi_{\mathrm{CEP}} \approx 3 \pi$ and $\Delta \phi_{\mathrm{CEP}} \approx 4 \pi$, we see a variation of the high-energy cutoff by more than $0.5 \mathrm{eV}$ (Fig. 3b). The variation of the low-energy cutoff is less pronounced and amounts to $0.2 \mathrm{eV}$. Plotting both quantities as a function of the CEP variation, clear out-of-phase oscillations and thus a narrowing and broadening of the spectrum with a period of $2 \pi$, that is, the oscillation period of the laser field, can be seen (Fig. 3c). The periodicity is confirmed by Fourier transforms (Fig. 3d) of the quantities plotted in Fig. 3c. Also, the total electron yield oscillates with the laser period, with a reduced modulation contrast of $10 \%$. The measurements present the first observation of CEP effects on the optical-field emission and near-field acceleration of electrons from an individual metallic nanostructure. Their generation and acceleration mechanism is distinctly different from the above-threshold photoemission studied previously ${ }^{4}$.

To account for these observations, we make use of the modified Simpleman model introduced above and study the effect of the CEP of a 2.6-cycle pulse at $1.65 \mu \mathrm{m}$ (Fig. 4a) on electron generation and motion. We describe the local near-field in the vicinity of the tip by $f \approx 9$ and a decay length of $2 \mathrm{~nm}$, values deduced experimentally. Of key importance are the acceleration of optical field-emitted electrons within spatial near-field gradients at the tip apex, and their dependence on the CEP and the birth time of the electron $\left(\Delta t_{\mathrm{B}}=t_{\mathrm{B}}-t_{0}\right)$ with respect to the negative amplitude maximum of the driving laser field at $t_{0}$. This acceleration is particularly pronounced for electrons emitted during the main cycle with maximum negative field amplitude.

Electrons with terminal kinetic energies close to the high-energy cutoff are emitted with birth times $-\pi / 2 \omega<\Delta t_{\mathrm{B}}<0$. Representative trajectories for $\Delta t_{\mathrm{B}}=-\pi / 2 \omega$ are shown in Fig. $4 \mathrm{~b}$. Evidently, these subcycle electrons are essentially accelerated only during the main half-cycle. They move along the field lines at the taper apex, escaping the near-field region before being back-accelerated during the subsequent positive half-cycle. Hence, their velocity remains constant at later times and their terminal kinetic energy is given by the near-field acceleration during the initial half-cycle. Recollisions, important for less sharp tips ${ }^{4}$, are strongly suppressed. Variation of the CEP affects the local field amplitude at the apex and therefore changes the terminal kinetic energy. A maximum energy is acquired for the highest field amplitude and thus for $\phi_{\mathrm{CEP}}=0$. Variation of the high-energy cutoff therefore mainly reflects the CEP-dependence of the strong-field acceleration of subcycle electrons within the spatial near-field gradient.

In the case of a spatially homogeneous driving field such as in conventional $\mathrm{HHG}$, electrons with $E_{\text {kin }} \approx 0$ are generated near $\Delta t_{\mathrm{B}}=0$. Here, the forward and backward acceleration during the positive and negative half-cycles effectively cancel out. Because of the large generation yield near $\Delta t_{\mathrm{B}}=0$, this results in the emission of a large number of slow electrons. CEP variations therefore mainly affect a small fraction of electrons with higher energy ${ }^{29}$, and lowenergy electrons are virtually unaffected. For sufficiently sharp tips, the strong near-field gradient breaks the symmetry between forward and backward acceleration. Accordingly, the slowest electrons are released for slightly positive $\Delta t_{\mathrm{B}} \approx 0.1 \pi / \omega$ (Fig. $4 \mathrm{c}$ ). The net acceleration of these electrons is mainly gained during the second cycle after their emission. Their kinetic energy therefore reflects the field amplitude during the subsequent negative halfcycle. This amplitude depends on the CEP of the laser, thus explaining the CEP effect on the low-energy electrons.

Simulations of CEP-dependent photoelectron spectra (Fig. 4d) confirm this scenario when assuming a sufficiently short decay length $(\sim 2 \mathrm{~nm})$. They account for the plateau-like shape of the 
spectra (Fig. 4e), revealing the dominant contribution of subcycle electrons to the emission process. The marked periodic modulation of the high-energy cutoff (Fig. 4f) reflects the variation of the maximum field amplitude at the tip surface with CEP.

These simulations suggest that electrons are released from a very small area $\left(\sim 10 \mathrm{~nm}^{2}\right)$ near the tip apex within a fraction of an optical cycle $(\sim 1 \mathrm{fs})$. One may therefore expect that space-charge effects $^{30}$ can influence the electron spectra and their CEP variation. Experimentally, we measured count rates of 1-3 electrons per pulse, indicating that a small number of electrons may be released within a single cycle. We tested space-charge effects in our simulations by allowing for the random generation of a finite number of electrons and by classically evaluating the repulsive forces among those electrons and their attraction to image charges (Supplementary Section 7). With an increasing number of electrons, we find a broadening and splitting of the kinetic energy spectra into two peaks, because the faster electrons are pushed forward by the cloud of slower electrons. Also, there is a clear increase in the angular distribution of the electron spectra because trajectories are becoming randomized by electron-electron collisions. Specifically, these simulations predict that the shape of the photoelectron spectra depends quite sensitively on the CEP. In contrast, in Fig. 3 we observe plateau-like spectra with a shape that is largely independent of the CEP. We take this as an indication that CEP effects in Fig. 3 are governed by phasedependent variations of the strong-field acceleration of subcycle electrons rather than by space-charge effects.

Taken together, our results present the first demonstration of CEP effects on the optical field emission of electrons from a single metallic nanostructure. The CEP controls the local amplitude of the near-field during the generation cycle and therefore the electron acceleration within the near-field gradient on a subcycle timescale. We believe that such field-driven control of the electron motion in the near-field of solid-state nanostructures can be seen as a new form of quantum electronics, paving the way towards the generation, measurement and application of attosecond electron pulses.

\section{Methods}

Tip fabrication. The single-crystalline gold tapers were fabricated from polycrystalline gold wires $(99.99 \%)$ with a diameter of $125 \mu \mathrm{m}$ (commercially available from Advent Research Materials). After cleaning in ethanol, the wires were annealed at $800^{\circ} \mathrm{C}$ for $8 \mathrm{~h}$ and then slowly (over another $8 \mathrm{~h}$ ) cooled to room temperature. These annealed wires were then electrochemically etched in $\mathrm{HCl}$ (aq. 37\%). For etching, rectangular voltage pulses with a frequency of $3 \mathrm{kHz}$ and a duty cycle of $10 \%$ were applied between the wire and a platinum ring serving as the counterelectrode. The tip shape was inspected by scanning electron microscopy.

Experimental set-up. Spectrally tunable, few-cycle near-infrared pulses with a stable and controllable CEP were generated by difference frequency generation of pulses from two NOPA stages ${ }^{26}$. Pulses from a Ti:sapphire regenerative amplifier (Newport, Spitfire Pro) with energy of $0.5 \mathrm{~mJ}$, duration of $120 \mathrm{fs}$, repetition rate of $1 \mathrm{kHz}$ and centre wavelength of $800 \mathrm{~nm}$ were split into three parts to form the pump sources for white light generation and, after frequency-doubling, for the two NOPA stages. White light was generated in a 2 -mm-thick sapphire plate and split into two parts, each part overlapping with a pump pulse in one the NOPA stages. Each stage consisted of a 1-mm-thick, type-I beta-barium borate (BBO) crystal cut to sustain amplification from $540 \mathrm{~nm}$ to $650 \mathrm{~nm}$, and from $870 \mathrm{~nm}$ to $890 \mathrm{~nm}$, respectively. Both output pulses were collinearly aligned and temporally overlapped in a 0.3 -mm-thick, type-II cut beta-barium borate (BBO) crystal to generate the difference frequency, resulting in passively CEP-stabilized pulses as short as $14 \mathrm{fs}$, tunable between $\lambda=1.3$ and $1.9 \mu \mathrm{m}$, and with a pulse energy of up to $220 \mathrm{~nJ}$. The CEP stability was checked in a conventional $f$-to- $2 f$ interferometer. The CEP of those pulses was controlled with a pair of fused silica wedges. A pulse pair with controllable inter-pulse delay was generated in a dispersion-balanced Michelson interferometer and focused onto the metal tips to a spot size of $0.6 \lambda / \mathrm{NA}$ by an all-reflective objective ${ }^{29}$ with a numerical aperture of $\mathrm{NA}=0.5$ under an angle of $90^{\circ}$ with respect to the tip axis. Electrons emitted from the tip were monitored simultaneously by a microchannel plate detector and a photoelectron spectrometer (Specs Phoibos 100, aligned along the tip axis) to measure their yield and kinetic energy spectra, respectively.

Data recording. To measure the electron yield, the microchannel plate was used and the laser intensity was varied by changing the arm length of the Michelson interferometer. The intensity scan was repeated 35 times, and the averaged curve is shown in Fig. 2a. To measure the CEP-dependent kinetic energy spectra, the photoelectron spectrometer detector was used and the CEP was controlled by wedge insertion. High-frequency noise from the NOPA was removed by using a zero-phase second-order Butterworth filter with a cutoff frequency of $3.5 \mathrm{eV}^{-1}$ (Fig. 2b).

Received 2 August 2013; accepted 26 September 2013; published online 10 November 2013; corrected online 19 November 2013

\section{References}

1. Herink, G., Solli, D. R., Gulde, M. \& Ropers, C. Field-driven photoemission from nanostructures quenches the quiver motion. Nature 483, 190-193 (2012).

2. Hommelhoff, P., Kealhofer, C. \& Kasevich, M. A. Ultrafast electron pulses from a tungsten tip triggered by low-power femtosecond laser pulses. Phys. Rev. Lett. 97, 247402 (2006).

3. Hommelhoff, P., Sortais, Y., Aghajani-Talesh, A. \& Kasevich, M. A. Field emission tip as a nanometer source of free electron femtosecond pulses. Phys. Rev. Lett. 96, 077401 (2006).

4. Kruger, M., Schenk, M. \& Hommelhoff, P. Attosecond control of electrons emitted from a nanoscale metal tip. Nature 475, 78-81 (2011).

5. Park, D. J. et al. Strong field acceleration and steering of ultrafast electron pulses from a sharp metallic nanotip. Phys. Rev. Lett. 109, 244803 (2012).

6. Ropers, C., Solli, D. R., Schulz, C. P., Lienau, C. \& Elsaesser, T. Localized multiphoton emission of femtosecond electron pulses from metal nanotips. Phys. Rev. Lett. 98, 043907 (2007).

7. Goulielmakis, E. et al. Attosecond control and measurement: lightwave electronics. Science 317, 769-775 (2007).

8. Aeschlimann, M. et al. Adaptive subwavelength control of nano-optical fields. Nature 446, 301-304 (2007)

9. Kubo, A. et al. Femtosecond imaging of surface plasmon dynamics in a nanostructured silver film. Nano Lett. 5, 1123-1127 (2005).

10. Stockman, M. I., Kling, M. F., Kleineberg, U. \& Krausz, F. Attosecond nanoplasmonic-field microscope. Nature Photon. 1, 539-544 (2007)

11. Goulielmakis, E. et al. Real-time observation of valence electron motion. Nature 466, 739-743 (2010).

12. Itatani, J. et al. Tomographic imaging of molecular orbitals. Nature 432, 867-871 (2004)

13. Barwick, B., Flannigan, D. J. \& Zewail, A. H. Photon-induced near-field electron microscopy. Nature 462, 902-906 (2009).

14. Zherebtsov, S. et al. Controlled near-field enhanced electron acceleration from dielectric nanospheres with intense few-cycle laser fields. Nature Phys. 7, 656-662 (2011)

15. Corkum, P. B. Plasma perspective on strong-field multiphoton ionization. Phys. Rev. Lett. 71, 1994-1997 (1993).

16. Haworth, C. A. et al. Half-cycle cutoffs in harmonic spectra and robust carrier-envelope phase retrieval. Nature Phys. 3, 52-57 (2007).

17. Popmintchev, T. et al. Bright coherent ultrahigh harmonics in the keV X-ray regime from mid-infrared femtosecond lasers. Science 336, 1287-1291 (2012).

18. Kulander, K. C., Schafer, K. J. \& Krause, J. L. Theoretical model for intense field high-order harmonic generation in rare gases. Laser Phys. 3, 359-364 (1993).

19. Bormann, R., Gulde, M., Weismann, A., Yalunin, S. V. \& Ropers, C. Tip-enhanced strong-field photoemission. Phys. Rev. Lett. 105, 147601 (2010).

20. Schenk, M., Krueger, M. \& Hommelhoff, P. Strong-field above-threshold photoemission from sharp metal tips. Phys. Rev. Lett. 105, 257601 (2010).

21. Baltuska, A. et al. Attosecond control of electronic processes by intense light fields. Nature 421, 611-615 (2003); erratum Nature 422, 189 (2003).

22. Nisoli, M. et al. Effects of carrier-envelope phase differences of few-opticalcycle light pulses in single-shot high-order-harmonic spectra. Phys. Rev. Lett. 91, 213905 (2003)

23. Eckle, P. et al. Attosecond angular streaking. Nature Phys. 4, 565-570 (2008).

24. Itatani, J. et al. Attosecond streak camera. Phys. Rev. Lett. 88, 173903 (2002).

25. Schmidt, S. et al. Adiabatic nanofocusing on ultrasmooth single-crystalline gold tapers creates a 10 -nm-sized light source with few-cycle time resolution. ACS Nano 6, 6040-6048 (2012).

26. Manzoni, C., Cerullo, G. \& De Silvestri, S. Ultrabroadband self-phase-stabilized pulses by difference-frequencygeneration. Opt. Lett. 29, 2668-2670 (2004)

27. Piglosiewicz, B. et al. Ultrasmall bullets of light-focusing few-cycle light pulses to the diffraction limit. Opt. Express 19, 14451-14463 (2011).

28. Stockman, M. I. \& Hewageegana, P. Absolute phase effect in ultrafast optical responses of metal nanostructures. Appl. Phys. A 89, 247-250 (2007).

29. Paulus, G. G. et al. Measurement of the phase of few-cycle laser pulses. Phys. Rev. Lett. 91, 253004 (2003).

30. Passlack, S. et al. Space charge effects in photoemission with a low repetition, high intensity femtosecond laser source. J. Appl. Phys. 100, 024912 (2006).

\section{Acknowledgements}

Financial support by the Deutsche Forschungsgemeinschaft (SPP1391), the European Union ('CRONOS') and the Korea Foundation for International Cooperation of Science 
and Technology (Global Research Laboratory project, K20815000003) is acknowledged. D.J.P. thanks Hanse-Wissenschaftskolleg for a personal fellowship. The research leading to these results has received funding from LASERLAB-EUROPE (grant agreement no. 284464, EC's Seventh Framework Programme).

\section{Author contributions}

C.L. initiated the project. C.M., P.F. and G.C. designed and implemented the laser system. B.P., S.S., J.V. and D.J.P. implemented the set-up and carried out the experiments. C.L., D.J.P., J.V. and P.G. developed the simulation model. D.J.P., P.G. and C.L. prepared the manuscript. All authors contributed to the final version of the manuscript.

\section{Additional information}

Supplementary information is available in the online version of the paper. Reprints and permissions information is available online at www.nature.com/reprints. Correspondence and requests for materials should be addressed to C.L.

\section{Competing financial interests}

The authors declare no competing financial interests. 